\title{
Calculation of Proton Passage through a Highly Filled Polyimide Composite
}

\author{
V. I. Pavlenko ${ }^{a}$, N. I. Cherkashina ${ }^{a, *, * *}$, and A. V. Noskov ${ }^{b}$ \\ ${ }^{a}$ Belgorod State Technological University, Belgorod, 308012 Russia \\ ${ }^{b}$ Belgorod State National Research University, Belgorod, 308015 Russia \\ *e-mail: cherkashina.ni@bstu.ru \\ **e-mail: natalipv13@mail.ru
}

Received January 14, 2020; revised February 25, 2020; accepted February 28, 2020

\begin{abstract}
The passage of protons in a polymer composite is studied by the calculation method. Composites based on polyimide and tungsten oxide are investigated. Data on their basic physical and mechanical properties are presented, depending on the filler content: density, ultimate tensile strength, water absorption, dielectric constant, linear thermal expansion coefficient. The introduction of the proposed filler, tungsten oxide, leads to improved physical and mechanical characteristics of the final composites. The ionization losses of protons in the composite under consideration are calculated for various contents of tungsten oxide in a wide energy range from 0.5 to $5 \mathrm{MeV}$. The filler is found to play a decisive role in estimating the average free path of protons. In the composites under consideration, the average free path of protons is small over the entire energy range. This indicates the possibility of using the composite to protect against proton radiation in outer space.
\end{abstract}

Keywords: cosmic radiation, polymer composite, radiation protection, ionization losses, proton range

DOI: $10.1134 /$ S 1027451020060129

\section{INTRODUCTION}

Cosmic radiation is one of the main problems in the implementation of interplanetary space flight, especially for manned missions beyond low Earth orbit. It can not only cause harm to the health of astronauts [1], but also damage the sensitive electronic equipment of spacecraft [2]. Cosmic radiation consists of various charged particles, including protons, electrons, and ions of heavy elements with energies and fluences in a wide range, which depend on the flight altitude, the solar cycle, and other factors [3, 4].

Galactic cosmic rays, sun rays, albedo particles and radiation belts are sources of cosmic radiation. The spectrum of galactic cosmic radiation in Earth's orbit consists of approximately $83 \%$ protons, $13 \%$ alpha particles, $3 \%$ electrons and $1 \%$ nuclei with $Z>2$. Solar cosmic rays are made up of protons, helium atoms and heavier ions. The protons of Earth's radiation belts make a huge contribution to cosmic radiation; taking them into account is especially important in the case of missions in low Earth orbit with an altitude of about $450 \mathrm{~km}[5,6]$.

Traditionally, metals and their alloys were used as protective shielding against radiation [7-9]. Currently, new composite materials are being actively developed, which not only have better radiation-protection properties compared to aluminum, the mate- rial that is currently used to create the majority of spacecraft structures, but also a lower mass [10-15].

The effectiveness of a potential shielding material is measured by its ability to attenuate the intensity of radiation as it passes through the material. However, when high-energy electrons and protons of cosmic radiation pass through heavy-metal materials, secondary radiation occurs, including charged particles, photons and neutrons, which does even more harm than the primary radiation $[16]$. In $[17,18]$ it was indicated that for some materials (aluminum and heavier elements) at certain thicknesses, the contribution of secondary particles to the total dose of the equivalent actually exceeds the contribution of primary particles. In this case, the use of lightweight materials for radiation shields, for example, polymers, is more efficient.

However, the use of pure polymers in space is limited due to their low thermal stability, low resistance to atomic oxygen, and a number of other negative factors of outer space [19-21]. In addition, the effect of radiation leads to the formation of new chemical bonds, which is usually accompanied by irreversible effects. These are manifested in a change in the appearance, chemical and physical states, as well as the mechanical, electrical, and thermal properties [22, 23]. The introduction of inorganic fillers into polymers allows the creation of composites with improved thermal, 
Table 1. Chemical composition of the developed highly filled composites

\begin{tabular}{c|c|c|c|c|c}
\hline \multirow{2}{*}{$\mathrm{WO}_{3}$ content, wt \% } & \multicolumn{5}{|c}{ Element content, at \% } \\
\cline { 2 - 6 } & $\mathrm{C}$ & $\mathrm{N}$ & $\mathrm{O}$ & $\mathrm{H}$ & $\mathrm{W}$ \\
\hline 0 & 68.11 & 7.57 & 21.62 & 2.70 & - \\
10 & 61.30 & 6.81 & 21.53 & 2.43 & 7.93 \\
20 & 54.49 & 6.05 & 21.44 & 2.16 & 15.86 \\
30 & 47.68 & 5.30 & 21.33 & 1.89 & 23.80 \\
40 & 40.86 & 4.55 & 21.25 & 1.62 & 31.72 \\
50 & 34.06 & 3.78 & 21.15 & 1.35 & 39.66 \\
60 & 27.24 & 3.03 & 21.06 & 1.08 & 47.59 \\
70 & 20.43 & 2.27 & 20.96 & 0.82 & 55.52 \\
80 & 13.62 & 1.51 & 20.87 & 0.54 & 63.46 \\
\hline
\end{tabular}

physical-mechanical and radiation-protection properties.

In this paper, we consider the effect of proton radiation as one of the most dangerous in cosmic radiation on highly filled polymer composites based on polyimide and tungsten(VI) oxide. The choice of a thermoplastic polyimide as the polymer matrix is due to its properties, which are necessary for use in outer space: high physical and mechanical properties at low and elevated temperatures; good anti-friction properties that are retained at low and high temperatures; high wear resistance and others [24, 25]. Resistance to proton radiation in the work is evaluated by calculation methods.

\section{MATERIALS AND METHODS}

As a polymer matrix, we used thermoplastic polyimide in the form of a molding compound of PI-PR20 brand (manufactured by G.S. Petrov Institute of Plastics). The dispersion of the polyimide particles was no more than 500 microns and the mass fraction of volatile substances was no more than $1 \%$. Tungsten (VI) oxide was used as a filler for the polyimide composites. The filler density was $7.4 \mathrm{~g} / \mathrm{cm}^{3}$ and the particle size was no more than 2 microns.

The composites were synthesized by direct pressing at a temperature of $380-400^{\circ} \mathrm{C}$ and a specific pressure of $500 \mathrm{kgf} / \mathrm{cm}^{2}$. The finished composite was a disk with a diameter of $30 \mathrm{~mm}$ and a thickness of 3-4 $\mathrm{mm}$. The content of tungsten oxide ranged from 10 to $80 \mathrm{wt} \%$. The composites were pressed using a Vaneox-40t automatic hydraulic press. The density of the obtained composites was determined by hydrostatic weighing. The tensile strength was determined on a UTS-10 universal mechanical testing machine.

The value of the thermal expansion was characterized by the temperature coefficient of linear expansion $\alpha$. The linear expansion coefficients of all the samples were measured at a temperature of from $20^{\circ} \mathrm{C}$ to $250^{\circ} \mathrm{C}$. The average value of $\alpha$ in the temperature range $\Delta T$ was determined by the formula:

$$
\alpha=\frac{\Delta l}{l} \frac{1}{\Delta T},
$$

where $\Delta l$ is the change in the sample length in the temperature range $\Delta T$, and $l$ is the initial size of the sample. The dielectric constant $\varepsilon$ was calculated based on the experimental value of the electrical capacity according to the formula:

$$
\varepsilon=\frac{C d}{S \varepsilon_{0}}
$$

where $C$ is the electrical capacity, $\varepsilon_{0}$ is the dielectric constant, $\varepsilon_{0}=8.85 \times 10^{-12} \mathrm{~F} / \mathrm{m}$, and $S$ is the area of the electrode (capacitor plate). The electrical capacity was determined using a Digital DT9205A multimeter using a flat capacitor.

To determine the water absorption of the finished composites, the samples were immersed in water. After 24 hours, the samples were removed from the water, moisture was removed from their surface with a soft damp cloth and immediately weighed. Water absorption was calculated by the formula:

$$
W=\left[\left(m_{2}-m_{1}\right) / m_{1}\right] \times 100 \%,
$$

where $m_{1}$ is the mass of the sample in the dry state [in g]; and $m_{2}$ is the mass of the sample after 24 hours of saturation with water [in g]. To calculate the passage of protons with energies from 0.5 to $5 \mathrm{MeV}$ in the developed highly filled composites, their atomic chemical composition was used (Table 1).

\section{RESULTS AND DISCUSSION}

Table 2 shows the main physical-mechanical properties of the obtained highly filled polyimide composites with tungsten (VI) oxide. Data analysis shows that the introduction of the proposed filler, $\mathrm{WO}_{3}$, leads to improved physical and mechanical characteristics of the final composites. The density of the pure sample of polyimide was $1.43 \mathrm{~g} / \mathrm{cm}^{3}$ and, upon the introduction of $80 \mathrm{wt} \% \mathrm{WO}_{3}$, the density increases three times to a value of $4.35 \mathrm{~g} / \mathrm{cm}^{3}$. The tensile strength of the composites initially significantly increases to a value of 157 MPa with a filler content of $60 \mathrm{wt} \%$, and then greatly reduced to $63 \mathrm{MPa}$. This indicates that with the introduction of more than $60 \mathrm{wt} \% \mathrm{WO}_{3}$ there is a lack of a binder polyimide for bonding into a single composite. Therefore, it is advisable to use composites with a content of the proposed filler of no more than $60 \mathrm{wt} \%$. Such a parameter as water absorption, when introduced, decreases significantly with increasing $\mathrm{WO}_{3}$. At the maximum $\mathrm{WO}_{3}$ content ( $80 \mathrm{wt} \%$ ) water absorption is $0.11 \%$, while the sample of pure polyimide absorbs moisture to a greater extent (water absorption $0.30 \%$ ). The linear expansion coefficients of all the samples were measured at a temperature from $20^{\circ} \mathrm{C}$ to 
Table 2. Physical-mechanical properties of the studied polyimide composites with tungsten(VI) oxide

\begin{tabular}{|c|c|c|c|c|c|c|c|c|c|}
\hline \multirow{2}{*}{ Parameter } & \multicolumn{9}{|c|}{$\mathrm{WO}_{3}$ content, wt $\%$} \\
\hline & 0 & 10 & 20 & 30 & 40 & 50 & 60 & 70 & 80 \\
\hline Density, $\mathrm{g} / \mathrm{cm}^{3}$ & 1.43 & 1.61 & 1.77 & 1.99 & 2.23 & 2.57 & 3.05 & 3.46 & 4.35 \\
\hline Tensile Strength, MPa & 77 & 89 & 103 & 119 & 132 & 145 & 157 & 121 & 63 \\
\hline Dielectric constant $1 \mathrm{kHz}$ & 3.5 & 5.7 & 7.8 & 9.8 & 11.6 & 13.5 & 15.1 & 17.2 & 19.2 \\
\hline Water absorption in 24 hours, $\%$ & 0.30 & 0.27 & 0.24 & 0.21 & 0.19 & 0.16 & 0.14 & 0.13 & 0.12 \\
\hline $\begin{array}{l}\text { Coefficient of linear expansion } \\
\text { from } 20^{\circ} \mathrm{C} \text { to } 250^{\circ} \mathrm{C}, \times 10^{-6}\end{array}$ & 4 & 4 & 3 & 3 & 3 & 3 & 2 & 2 & 2 \\
\hline
\end{tabular}

$250^{\circ} \mathrm{C}$. The coefficients of all the studied composites are low (Table 2).

Based on the data in Table 2, it can be argued that the developed composites based on polyimide and tungsten (VI) oxide have high physical and mechanical characteristics and can be used in the field of space materials science. To assess the possibility of using the developed materials in the space industry, it is necessary to study the stability of these materials to various types of ionizing radiation present in outer space. The following are the results of evaluating the radiationprotection properties of composites with respect to proton radiation using mathematical modeling. This method allows one to make an initial prediction of the stability of materials to the effects of protons without conducting expensive experiments on the irradiation of materials in space or proton accelerators.

We use the Bethe-Bloch formula for the linear stopping power [in $\mathrm{MeV} / \mathrm{cm}$ ]. In the case of a low kinetic energy of protons $\left(E_{k} \ll M_{p} c^{2}\left(\frac{M_{p} c^{2}}{2 m_{e} c^{2}}-1\right)\right)$ it takes the form:

$$
\begin{gathered}
\left(-\frac{d E}{d x}\right)_{\mathrm{col}}=K \rho \frac{z^{2} Z}{2 A} \frac{1}{2 \beta^{2}} \\
\times\left[2 \ln \left(\frac{2 m_{e} c^{2} \beta^{2}}{I\left(1-\beta^{2}\right)}\right)-2 \beta^{2}-U-\delta\right],
\end{gathered}
$$

where $U$ is the correction for taking into account electron bonding at the $K$ - and $L$ shells, and $\delta$ takes into account the polarization effect of the medium (density effect). In the problem under consideration $U$ and $\delta$ are not significant. The following notation is used in the formula: $\beta=\sqrt{1-\frac{\left(M_{p} c^{2}\right)^{2}}{\left(M_{p} c^{2}+E_{k}\right)^{2}}}$ is the Lorenz factor of a proton with the kinetic energy $E_{k}, K=$ $4 \pi r_{e}^{2} m_{e} c^{2} N_{\mathrm{A}}=0.307 \mathrm{MeV} \mathrm{g}^{-1} \mathrm{~cm}^{2}, m_{e} c^{2}=0.511 \mathrm{MeV}$ is the rest energy of an electron, $M_{R} c^{2}=938.3 \mathrm{MeV}$ is the proton rest energy, $Z$ is the particle charge in units of positron charge (for a proton $Z=1$ ), $r_{e}=\frac{e^{2}}{m_{e} c^{2}}=2.8 \times$ $10^{-13} \mathrm{~cm}$ is the classical radius of an electron, $N_{\mathrm{A}}=6 \times$ $10^{23} \mathrm{~mol}^{-1}, \rho$ is the density of the substance, and $I$ is the average ionization potential of an atom of the substance in the medium.

The composite material under study consists of atoms of various types, each of which will contribute to ionization loss of the electron energy. We use Bragg's composition law:

$$
\left(-\frac{d E}{d x}\right)_{\text {col }}=\sum_{i} \rho_{i}\left(-\frac{d E}{d x}\right)_{i}
$$

where $\rho_{i}$ and $\left(-\frac{d E}{d x}\right)_{i}$ are the density and contribution of the $i$ th element in a complex substance to the ionization loss of a proton.

We rewrite expression (5) in a more convenient form for analysis:

$$
\begin{gathered}
\left(-\frac{d E}{d x}\right)_{\mathrm{col}}=\rho \frac{Z}{A} F_{p}\left(E_{k}, I\right), \\
F_{p}\left(E_{k}, I\right)=\frac{K}{\beta^{2}}\left[2 \ln \left(\frac{2 m_{e} c^{2} \beta^{2}}{I\left(1-\beta^{2}\right)}\right)-\beta^{2}\right] .
\end{gathered}
$$

In this case, the contribution of each substance to the total ionization loss has the form:

$$
\begin{aligned}
& \left(-\frac{d E}{d x}\right)_{\mathrm{col}}^{\mathrm{C}}=\rho_{\mathrm{C}} \frac{Z_{\mathrm{C}}}{A_{\mathrm{C}}} F_{p}\left(E_{k}, I_{\mathrm{C}}\right), \\
& \left(-\frac{d E}{d x}\right)_{\mathrm{col}}^{\mathrm{N}}=\rho_{\mathrm{N}} \frac{Z_{\mathrm{N}}}{A_{\mathrm{N}}} F_{p}\left(E_{k}, I_{\mathrm{N}}\right), \\
& \left(-\frac{d E}{d x}\right)_{\mathrm{col}}^{\mathrm{O}}=\rho_{\mathrm{O}} \frac{Z_{\mathrm{O}}}{A_{\mathrm{O}}} F_{p}\left(E_{k}, I_{\mathrm{O}}\right), \\
& \left(-\frac{d E}{d x}\right)_{\mathrm{col}}^{\mathrm{H}}=\rho_{\mathrm{H}} \frac{Z_{\mathrm{H}}}{A_{\mathrm{H}}} F_{p}\left(E_{k}, I_{\mathrm{H}}\right), \\
& \left(-\frac{d E}{d x}\right)_{\mathrm{col}}^{\mathrm{W}}=\rho_{\mathrm{W}} \frac{Z_{\mathrm{W}}}{A_{\mathrm{W}}} F_{p}\left(E_{k}, I_{\mathrm{W}}\right),
\end{aligned}
$$




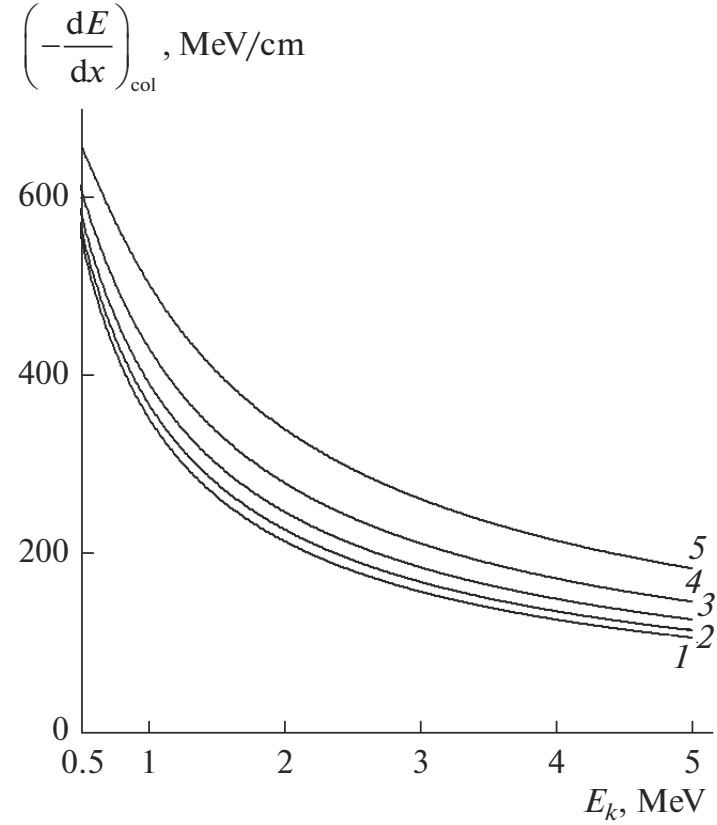

Fig. 1. Curves of the dependence of the ionization losses of the composites on the initial energy of protons at different $\mathrm{WO}_{3}$ contents: 0 (1); 20 (2); 40 (3); 60 (4); 80 wt \% (5).

The average ionization potentials of the atoms in question have the following meanings: $I_{\mathrm{C}} \approx 78, I_{\mathrm{N}} \approx 82$, $I_{\mathrm{O}} \approx 95, I_{\mathrm{H}} \approx 19.2, I_{\mathrm{W}} \approx 727 \mathrm{eV}$.

Based on Bragg's composition law (5), we write the ionization losses of the proton energy in the studied composite material in the form:

$$
\begin{gathered}
\left(-\frac{d E}{d x}\right)_{\text {col }}=\rho_{\mathrm{C}} \frac{Z_{\mathrm{C}}}{A_{\mathrm{C}}} F_{p}\left(E_{k}, I_{\mathrm{C}}\right) \\
+\rho_{\mathrm{N}} \frac{Z_{\mathrm{N}}}{A_{\mathrm{N}}} F_{p}\left(E_{k}, I_{\mathrm{N}}\right)+\rho_{\mathrm{O}} \frac{Z_{\mathrm{O}}}{A_{\mathrm{O}}} F_{p}\left(E_{k}, I_{\mathrm{O}}\right) \\
+\rho_{\mathrm{H}} \frac{Z_{\mathrm{H}}}{A_{\mathrm{H}}} F_{p}\left(E_{k}, I_{\mathrm{H}}\right)+\rho_{\mathrm{W}} \frac{Z_{\mathrm{W}}}{A_{\mathrm{W}}} F_{p}\left(E_{k}, I_{\mathrm{W}}\right) .
\end{gathered}
$$

Figure 1 shows the curves constructed by formulas (7) and (9). They demonstrate the ionization loss of the obtained polymer composite for different $\mathrm{WO}_{3}$ contents in the composite. Analysis of the figure showed that with an increase in the content of $\mathrm{WO}_{3}$ ionization losses increase at the same initial proton energy. The smallest ionization loss is characterized by the sample without the filler, and the largest, the composite with a filler content of $80 \mathrm{wt} \%$.

One of the radiation-protection characteristics is the mean free path of protons in the material. This is calculated by the formula

$$
R\left(E_{0}\right)=\int_{0}^{E_{0}} \frac{d E_{k}}{\left(-\frac{d E}{d x}\right)_{\mathrm{col}}} .
$$

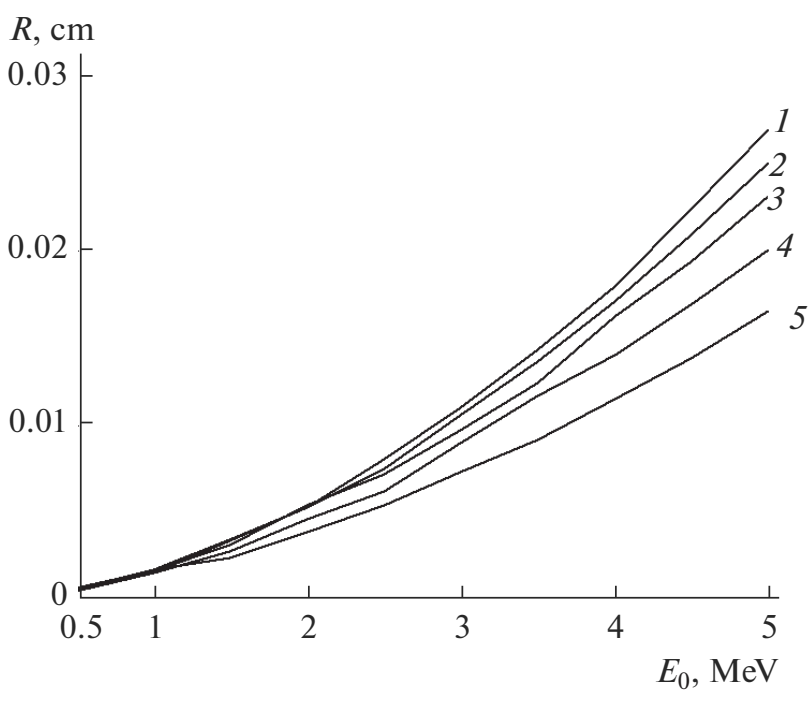

Fig. 2. Curves of the dependence of the mean free path of protons in the composites on their initial energy at different $\mathrm{WO}_{3}$ contents: 0 (1); 20 (2); 40 (3); 60 (4); 80 wt \% (5).

Figure 2 presents curves demonstrating the dependence of the mean free path of an electron in the composite on its initial kinetic energy, for different $\mathrm{WO}_{3}$ contents in the composite. Analysis of the figure showed that with a higher filler concentration, the mean free path decreases (at the same proton energy). That is, the filler plays a decisive role in estimating the mean free path of protons. The data in Fig. 2 show that in the composites under consideration the mean free path of protons is small over a wide range of initial energies $(1-5 \mathrm{MeV})$. This indicates the possibility of using the composite for protection against proton radiation in outer space. For comparison, Fig. 3 presents data on the average ranges of protons with an energy of $5 \mathrm{MeV}$ in a composite containing $\mathrm{WO}_{3} 80 \mathrm{wt}$

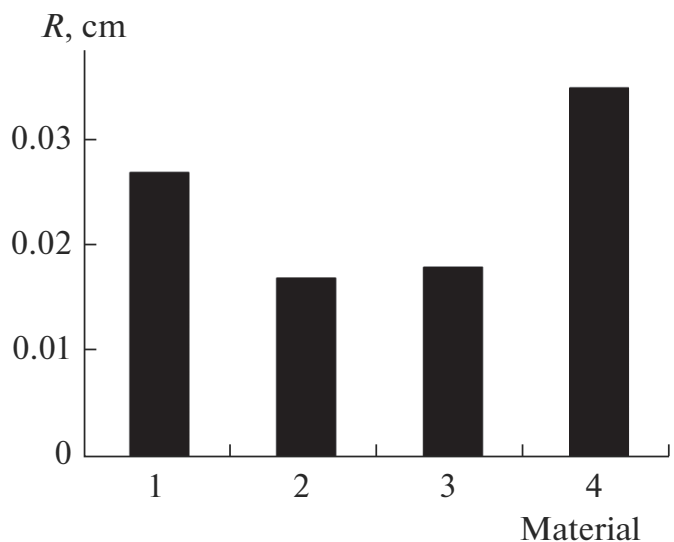

Fig. 3. Mean free path of $2-\mathrm{MeV}$ protons in various materials: 1 - polyimide; 2 - composite $\left(80\right.$ wt $\left.\% \mathrm{WO}_{3}\right) ; 3-$ aluminum; 4 - polystyrene. 
$\%$, aluminum and polymers [26]. The effective mean free path of protons in the studied composite is close to that in aluminum. The path of protons in polymers without a filler is almost two times greater than in the composite under consideration.

\section{CONCLUSIONS}

The work found that the introduction of the proposed filler, $\mathrm{WO}_{3}$, leads to improved physical and mechanical characteristics of the final composites. The density of the pure sample of polyimide is $1.43 \mathrm{~g} / \mathrm{cm}^{3}$, and upon the introduction of $80 \mathrm{wt} \%$ $\mathrm{WO}_{3}$ the density increases three times to $4.35 \mathrm{~g} / \mathrm{cm}^{3}$. The linear thermal expansion coefficients of all the studied composites are small and do not exceed the value $2 \times 10^{-6}$. It is shown that it is advisable to use composites with a filler content of no more than $60 \mathrm{wt} \%$, since at a higher concentration, the strength characteristics decrease.

Calculated data on the passage of protons through the studied composites are presented. With increasing content of $\mathrm{WO}_{3}$, the ionization losses increase at the same initial proton energy. The smallest ionization loss is characterized by the sample without the filler, and the largest, by the composite with a filler content of $80 \mathrm{wt} \%$.

It was found that the filler plays a decisive role in estimating the mean free path of protons. In the composites under consideration, the mean free path of protons is small over a wide range of initial energies $(1-5 \mathrm{MeV})$. This indicates the possibility of using the composite for protection against proton radiation in outer space. The effective mean free path of protons in the studied composite is close to that in aluminum. The path of protons in polymers without a filler is almost two times greater than in the composite under consideration.

\section{ACKNOWLEDGMENTS}

The study was supported by the Russian Science Foundation (grant no. 19-19-00316).

\section{REFERENCES}

1. J. C. Chancellor, G. B. I. Scott, and J. P. Sutton, Life 4 (3), 491 (2014). https://doi.org/10.3390/life4030491

2. S. Duzellier, Aerosp. Sci. Technol. 9 (1), 93 (2005). https://doi.org/10.1016/j.ast.2004.08.006

3. V. D. Kuznetsov, Phys.-Usp. 55, 305 (2012).

4. V. D. Kuznetsov, Kosm. Tekh. Tekhnol., No. 3 (6), 3 (2014).

5. Managing Space Radiation Risk in the New Era of Space Exploration (National Academic, Washington D.C., 2008), p. 27.
6. V. L. Ginsburg, Phys.-Usp. 44, 1107 (2001).

7. N. Read, W. Wang, K. Essa, and M. M. Attallah, Mater. Des. 65, 417 (2015). https://doi.org/10.1016/j.matdes.2014.09.044

8. R. Rioja and J. Liu, Metall. Mater. Trans. A 43, 3325 (2012). https://doi.org/10.1007/s11661-012-1155-z

9. C. S. Kumar, S. M. Mayanna, K. N. Mahendra, et al., Appl. Surf. Sci. 151, 280 (1999). https://doi.org/10.1016/S0169-4332(99)00290-1

10. L. Dong, J. Ko, and J. -K. Woo, New Phys.: Sae Mulli 64, 1248 (2014). https://doi.org/10.3938/NPSM.64.1248

11. R. H. Khasanshin, L. S. Novikov, and S. B. Korovin, J. Surf. Invest.: X-ray, Synchrotron Neutron Tech. 9, 81 (2015). https://doi.org/10.1134/S1027451015010115

12. V. I. Pavlenko, N. I. Cherkashina, and D. A. Ivanitskii, Vestn. Belgorod. Gos. Tekh. Univ. im. V.G. Shukhova, No. 10, 185 (2016).

13. T. Bel, C. Arslan, and N. Baydogan, Mater. Chem. Phys. 221, 58 (2019). https://doi.org/10.1016/j.matchemphys.2018.09.014

14. F. E. Vilkov, A. A. Lozovan, A. V. Bazhanov, et al., J. Surf. Invest.: X-ray, Synchrotron Neutron Tech. 11, 912 (2017). https://doi.org/10.1134/S1027451017050160

15. K. Rojdev and W. Atwell, Gravit. Space Res 3, 59 (2015).

16. A. Keating, P. Goncalves, M. Pimenta, et al., Radiat. Environ. Biophys. 51 (3), 245 (2012). https://doi.org/10.1007/s00411-012-0412-2

17. I. P. Bezrodnykh, E. I. Morozova, and A. A. Petrukovich, Vopr. Elektromekh. 120, 37 (2011).

18. Model of Space, Vol. 2, Impact of the Space Environment on the Spacecraft Materials and Equipment, Ed. by L. S. Novikov (Mosk. Gos. Univ., Moscow, 2007) [in Russian].

19. N. I. Cherkashina, V. I. Pavlenko, and A. V. Noskov, Radiat. Phys. Chem. 159, 117 (2019). https://doi.org/10.1016/j.radphyschem.2019.02.041

20. L. Zhang and R. Chen, Chin. J. Aeronaut 17, 53 (2004). https://doi.org/10.1016/S1000-9361(11)60203-3

21. Z. Hooshangi, S. A. H. Feghhi, and R. Saeedzadeh, Acta Astronaut. 119, 233 (2016). https://doi.org/10.1016/j.actaastro.2015.11.031

22. Z. Kacarevic-Popovic, D. Kostoski, L. Novakovic, et al., J. Serb. Chem. Soc. 69, 1029 (2004).

23. A. Faltermeier, C. Reicheneder, P. Romer, et al., J. Orofac. Orthop. 75 (5), 334 (2014). https://doi.org/10.1007/s00056-014-0229-5

24. X. Fang, Z. Yang, S. Zhang, et al., Polymer 45, 2539 (2004). https://doi.org/10.1016/j.polymer.2004.02.008

25. S. Xie, Z. Zhang, and W. Wei, J. Korean Phys. Soc. 51, $1536(2007)$. https://doi.org/10.3938/jkps.51.1536

26. P. A. Ponomarenko and M. A. Frolova, Global. Yad. Bezop., No. 4, 90 (2015). 